\title{
Point of care diaphragmatic ultrasonography made easy
}

\author{
Kariem El-Boghdadly, MBBS $($ D $\cdot$ Alberto Goffi, MD • Vincent Chan, MD
}

Received: 10 October 2016/Revised: 11 October 2016/Accepted: 21 October 2016/Published online: 28 October 2016

(C) Canadian Anesthesiologists' Society 2016

\section{To the Editor,}

More than half a million ambulatory shoulder operations $^{1}$ and 50,000 shoulder arthroplasties are performed annually in the United States alone, with an ever-expanding frequency. ${ }^{2}$ A common side effect of interscalene brachial plexus block (ISB) performed for shoulder surgery is transient phrenic nerve palsy (up to a $100 \%$ incidence), ${ }^{3}$ which may have significant clinical implications in patients with obstructive sleep apnea, obesity, and/or pulmonary pathology.

Recently, Naik et al. described a novel ultrasonographic approach using a high-frequency linear probe to evaluate diaphragmatic function by assessing the motion and thickness of the diaphragm during the sniff test. ${ }^{4}$ The purported advantage of this test is the ease of imaging the muscular portion of the diaphragm and measuring the amplitude of diaphragmatic movement in both the B- and M-modes, independent of hepatic and splenic acoustic windows. The results are thus potentially more reproducible.

We wish to further simplify diaphragm ultrasonography to make it more practical and accessible to the everyday practitioner using simple surface marking. Similar to the Naik et al. concept, we place a high-frequency (10-15 $\mathrm{MHz}), 38-\mathrm{mm}$ linear array probe in the coronal plane over

Electronic supplementary material The online version of this article (doi:10.1007/s12630-016-0766-z) contains supplementary material, which is available to authorized users.

K. El-Boghdadly, MBBS ( $\square)$

Guy's \& St Thomas' NHS Foundation Trust, London, UK

e-mail: elboghdadly@gmail.com

A. Goffi, MD · V. Chan, MD

Toronto Western Hospital, Toronto, ON, Canada the mid-axillary line and set the initial field depth to $3-4 \mathrm{~cm}$ (Figure). The starting probe level is in the vicinity of the seventh to eighth ribs (R7-R8) on the right side and the eighth to ninth ribs (R8-R9) on the left side, with the cephalad orientation to the left of the screen. The homogeneous, grey appearance of the liver on the right or with the spleen on the left should be centered, with the acoustic shadowing of the ribs on either side. The diaphragm is noted superficial to these two visceral organs and deep to the intercostal muscles. The patient is then asked to take a deep breath with the probe in situ, at which point we can observe the caudal descent of the liver or spleen (toward the right of the screen), commonly over two intercostal spaces. The hyperechoic pleural line is visualized between the ribs, cephalad to the liver or spleen. The gross caudal movement of the pleural line can be easily followed as a qualitative assessment of diaphragmatic excursion. Additionally, the actual pleural line position can be marked externally on the skin at the end of a full expiration (minimum distance, Figures A, B) and inspiration (maximum distance, Figures $\mathrm{C}, \mathrm{D}$ ). The difference between these two points provides a quantitative measure of the diaphragmatic excursion (video; available as Electronic Supplementary Material). To avoid having the rib acoustic shadow block the pleural line view, the probe can be turned slightly obliquely to align it over the intercostal space to show the transition from the pleural line to the diaphragm (zone of apposition) (Figures E, F).

We perform diaphragmatic evaluations before and after interscalene block using this simple method. A minimal change in the distance that the diaphragm moves between expiration and inspiration indicates the presence of phrenic nerve palsy after ISB (Figures G, H). The positional change can be confirmed qualitatively and quantitatively (\% change). 


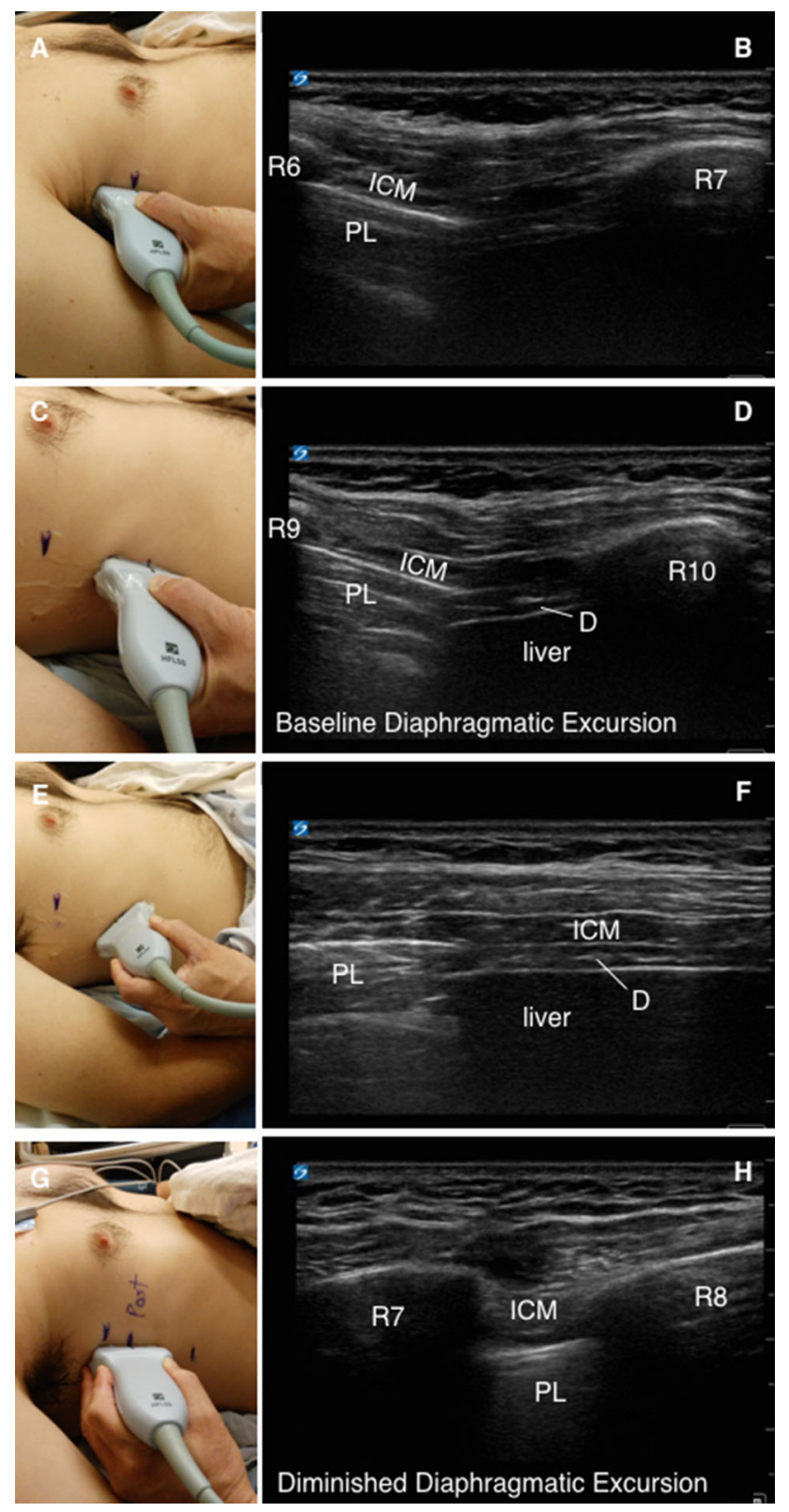

Figure A. Linear probe position at the right mid-axillary line at the end of full expiration (pre-block). B. Corresponding sonogram at the end of full expiration (pre-block). C. Linear probe position at the right mid-axillary line at the end of full inspiration (pre-block). D. Corresponding sonogram at the end of full inspiration (pre-block). E. Right oblique linear probe position at the end of full inspiration (preblock). F. Corresponding sonogram at the end of full inspiration (preblock). G. Linear probe position at the right mid-axillary line at the end of full inspiration (post-block). H. Corresponding sonogram at the end of full inspiration (post-block)

This simple technique is quick, is easy to learn, and can be performed on both the right and left sides. We hope that this surface method for assessing diaphragmatic function will increase the likelihood of identifying phrenic nerve palsy following ISB. Validation of this promising method vs well-established techniques ${ }^{5}$ (e.g., M-mode diaphragmatic excursion measurement using a curved probe) is now needed.

Conflicts of interest None declared.

Editorial responsibility This submission was handled by Dr. Hilary P. Grocott, Editor-in-Chief, Canadian Journal of Anesthesia.

Financial sources supporting the work None.

\section{References}

1. Jain NB, Yamaguchi $K$. The contribution of reverse shoulder arthroplasty to utilization of primary shoulder arthroplasty. J Shoulder Elb Surg 2014; 23: 1905-12.

2. Kim SH, Wise BL, Zhang Y, Szabo RM. Increasing incidence of shoulder arthroplasty in the United States. J Bone Joint Surg Am 2011; 93: 2249-54.

3. Urmey WF, Talts $K H$, Sharrock NE. One hundred percent incidence of hemidiaphragmatic paresis associated with interscalene brachial plexus anesthesia as diagnosed by ultrasonography. Anesth Analg 1991; 72: 498-503.

4. Naik LY, Sondekoppam RV, Tsui JJ, Tsui BC. An ultrasoundguided $\mathrm{ABCDE}$ approach with a sniff test to evaluate diaphragmatic function without acoustic windows. Can J Anesth 2016; 63: 1199-200.

5. Boussuges A, Gole Y, Blanc P. Diaphragmatic motion studied by M-mode ultrasonography: methods, reproducibility, and normal values. Chest 2009; 135: 391-400. 